\title{
Transient Analysis of Silicon Devices Using the Hydrodynamic Model
}

\author{
LUIGI COLALONGO*, MARINA VALDINOCI, ANTONIO GNUDI and MASSIMO RUDAN
}

Dipartimento di Elettronica, Informatica e Sistemistica, Università di Bologna, Viale Risorgimento 2, 40136 Bologna, Italy

The analysis of the switching behaviour of submicron devices brings about the necessity of extending the solution of the hydrodynamic model to the transient case. The implementation of such model has been carried out and a few examples of simulation are presented here, showing the velocity-overshoot of a ballistic diode and the temperature spread in the drain region of a realistic MOS device.

Keywords: Submicron Devices, Hydrodynamic Model, Transient Analysis, Velocity-Overshoot, Numerical Methods, Semiconductor-Device Simulation.

\section{MATHEMATICAL MODEL}

In recent years increasing attention has been paid to the analysis of the switching behaviour of submicron devices, bringing about the necessity of extending the solution of the Hydrodynamic Model (HD) to the transient case $[1,2]$. The Hydrodynamic Model in the transient case reads [3]:

$$
\begin{gathered}
-\operatorname{div}\left(\varepsilon_{s} \operatorname{grad} \varphi\right)=q\left(p-n+N_{D}^{+}-N_{A}^{-}\right), \\
\partial n / \partial t-\operatorname{div}\left(\mathbf{J}_{n} / q\right)=-U, \quad \partial p / \partial t+\operatorname{div}\left(\mathbf{J}_{p} / q\right)=U,
\end{gathered}
$$

$$
\partial\left(n w_{n}\right) / \partial t+\operatorname{div}\left[-\kappa_{n} \operatorname{grad} T_{n}-(5 / 2) k_{B} T_{n} \mathbf{J}_{n} / q\right]
$$$$
=\mathbf{E} \bullet \mathbf{J}_{n}-w_{n} U-n\left(w_{n}-w_{n o}\right) / \tau_{w n}
$$

$$
\partial\left(p w_{p}\right) / \partial t+\operatorname{div}\left[-\kappa_{p} \operatorname{grad} T_{p}+(5 / 2) k_{B} T_{p} \mathbf{J}_{p} / q\right]
$$

$$
=\mathbf{E} \bullet \mathbf{J}_{p}-w_{p} U-p\left(w_{p}-w_{p o}\right) / \tau_{w p}
$$

where

$$
\begin{gathered}
\mathbf{J}_{n}=q D_{n} \operatorname{grad} n+q \mu_{n} n \operatorname{grad}\left(k_{B} T_{n} / q-\varphi\right), \\
\mathbf{J}_{p}=-q D_{p} \operatorname{grad} p+q \mu_{p} p \operatorname{grad}\left(k_{B} T_{p} / q-\varphi\right)
\end{gathered}
$$

and $T, \tau_{p}, \tau_{w}$ are the temperature, momentum-relaxation time, and energy-relaxation time of the carriers, and $w=(1 / 2) m v^{2}+(3 / 2) k_{B} T$ is the average kinetic energy. The remaining symbols have the usual meaning. This set of equations is discretized in space using the so-called Box Integration Method. The time derivatives are discretized using the Gear method which is an $A$-stable, $L$-stable method for stiff differential equations. The discretization yields at each time step a system of $5 N$ algebraic equations, where $N$ is the size of the discretization grid [3]. The system of nonlinear equations is solved using the following scheme: first, the system of Poisson, electron- and hole-continuity equations is solved by a coupled Newton method, then the temperature is updated solving the energy-balance equations for electrons and holes. The

* Corresponding author. Tel: +39 (51) 644-3016. Fax: +39 (51) 644-3073. E-mail: Icolalongo@deis.unibo.it 
external loop is repeated until the global convergence is reached, and the whole procedure is repeated at each time step. The solution scheme depicted above is easier to implement than the full Newton method coupling all equations; it has proven very stable and, supplemented with a quadratic projection algorithm, rather fast as well. The term $E \cdot J$ is treated by the vectorial identity $E \cdot J=-\operatorname{div}(\varphi J)+\varphi \operatorname{div}(J)$ [4]. In steady-state conditions the term $\operatorname{div}(\mathbf{J})$ at the RHS is often replaced by $q U$ using the continuity equations; in transient conditions this is no longer possible because of the time derivatives. Straightforward manipulations lead to the following discretized expression (referring to the $i^{\text {th }}$ node):

$$
\Omega_{i}[\mathbf{E} \bullet \mathbf{J}]_{i}=\sum_{j \neq i} \frac{d_{i j} J_{i j}}{2}\left(\varphi_{i}-\varphi_{j}\right)
$$

where $d_{i j}$ is the normal bisector of the triangle side and $\Omega_{i}$ is the area of the node. This expression is easy to handle as the time dependence is not explicit: therefore, in the transient case there is no need to account for the time derivatives in $\operatorname{div}(\boldsymbol{J})$ and $\operatorname{div}(\varphi \boldsymbol{J})$.

\section{RESULTS}

The simulations have been carried out using a twodimensional version of the device-analysis program HFIELDS [3], supplemented with the method desscribed above, first on a $0.5 \mu m, n^{+}-n-n^{+}$ballistic diode having a $0.35 \mu \mathrm{m} n$-region. The diode is initially at equilibrium, then it is biased by a linear ramp which brings the voltage drop between the contacts from 0 to $2 \mathrm{~V}$ in $1 \mathrm{psec}$; the voltage on this contact remains at $2 \mathrm{~V}$ for $1 \mu \mathrm{sec}$ after the end of the ramp. It is worth noting that the duration of the ramp is about one order of magnitude larger than the carrier momentum-relaxation time; for this reason the terms $\partial J / \partial t$ are dropped from the transport equations. On the contrary, the time derivatives are retained in $(3,4)$ because the carrier energy-relaxation times are larger [5]. In any case the ramp is slow enough to allow for the steady-state approximation leading to Poisson's equation. Fig. 1 shows the normalized temperature at two different times 1 psec and $1 \mu \mathrm{sec}$. The first one is

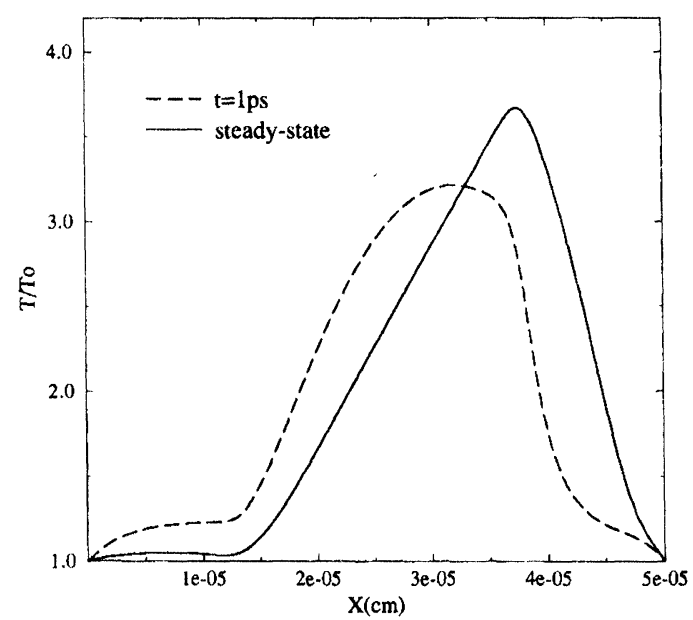

FIGURE 1 Ballistic diode. Normalized temperature at two different times: $t=1 \mathrm{psec}$ (end of the ramp) and $t=1 \mu \mathrm{sec}$ (transient extinguished)

of the same order as $\tau_{w}$ and corresponds to the instant at which the ramp reaches $2 \mathrm{~V}$; at $t=1 \mu \mathrm{sec}$ the transient is extinguished. The figure exhibits the delay in the temperature growth due to the time derivatives in $(3,4)$. Physically this means that the energy cannot change immediately after the switching even if the temperature changes considerably faster with respect to the other physical quantities (electron concentration, electric field). In Fig. 2 a velocity overshoot is

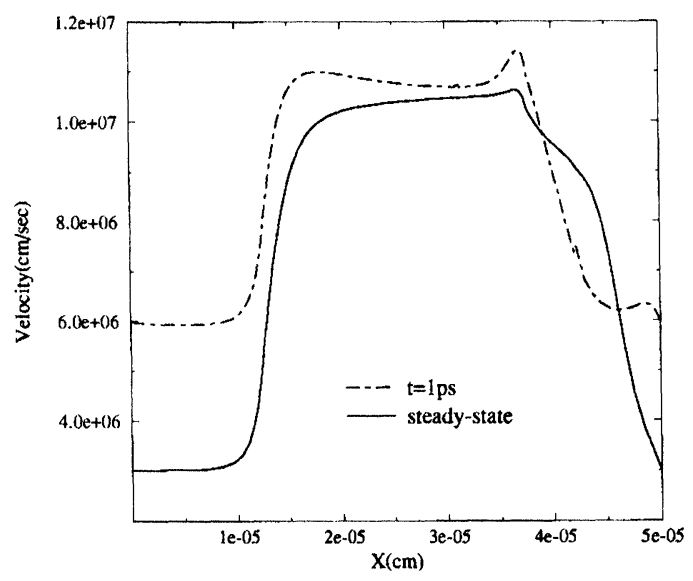

FIGURE 2 Ballistic diode. Carrier velocity at $t=1 \mathrm{psec}$ and $t=$ $1 \mu \mathrm{sec}$. The velocity overshoot is observed at $t=1 \mathrm{psec}$ 


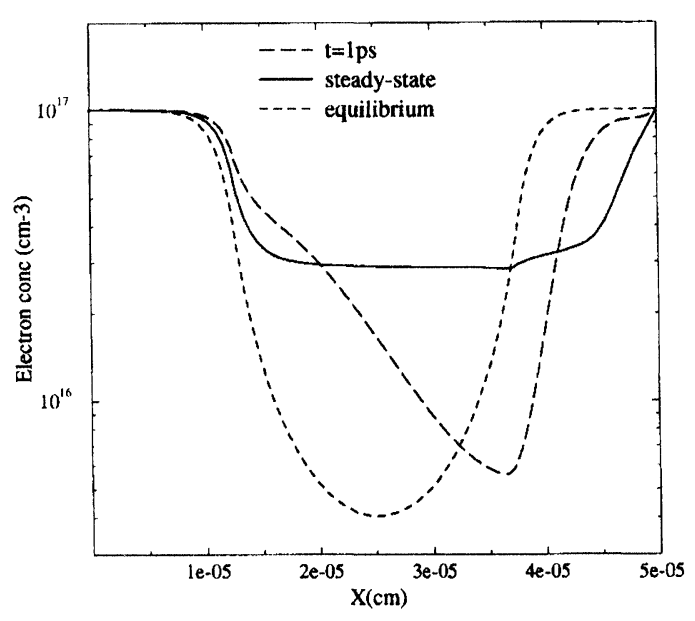

FIGURE 3 Ballistic diode. Electron concentration at equilibrium, at $t=1 \mathrm{psec}$ and at $t=1 \mu \mathrm{sec}$

observed at $t=1 \mathrm{psec}$, due to the fact that the momentum-relaxation time is smaller by about one order of magnitude than the energy-relaxation time. Because of this, the velocity increase is considerably faster than that of energy, as is seen in Figs. 1 and 2. As the carrier temperature grows, the velocity overshoot becomes less important. The features of the momentum and energy-relaxation times are reflected into the mobility model used in the HD equations, $\mu(w)=\mu_{o} /\left[1+\alpha\left(w-w_{o}\right)\right], \alpha=\mu_{o} /\left(q \tau_{w} v_{\text {sat }}^{2}\right)$ : in the first time intervals $\left(t<\tau_{w}\right)$, while the temperature is

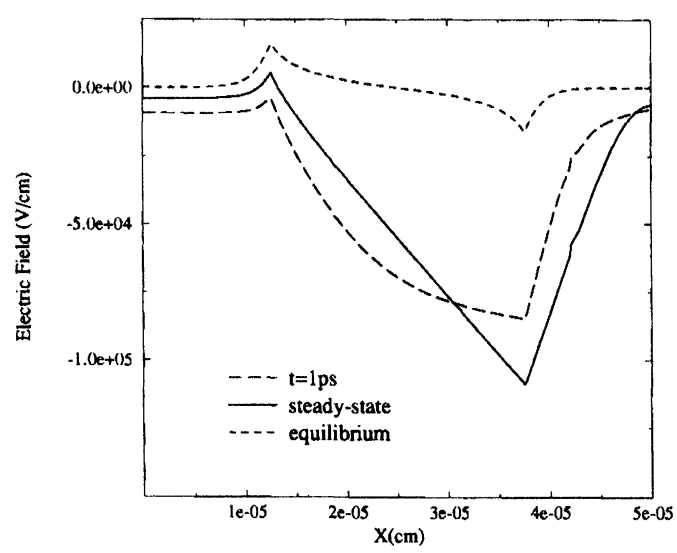

FIGURE 4 Ballistic diode. Electric field at equilibrium, at $t=1$ psec and at $t=1 \mu \mathrm{sec}$ still growing the mobility is in fact larger than in steady-state conditions. Figs. 3 and 4 show the electron concentration and the electric field respectively at the same time intervals and at equilibrium. Next, more simulations have been carried out on a realistic $n$-type, $0.7 \mu \mathrm{m}$ channel MOS transistor. The source and gate voltages were set at 0 and $3 \mathrm{~V}$, respectively. Starting from equilibrium, the drain voltage was first brought from 0 to $3 \mathrm{~V}$ using a 1-psec linear ramp, then kept at $3 \mathrm{~V}$ for $1 \mu \mathrm{sec}$. Figs. 5, 6 and 7 show the nor-

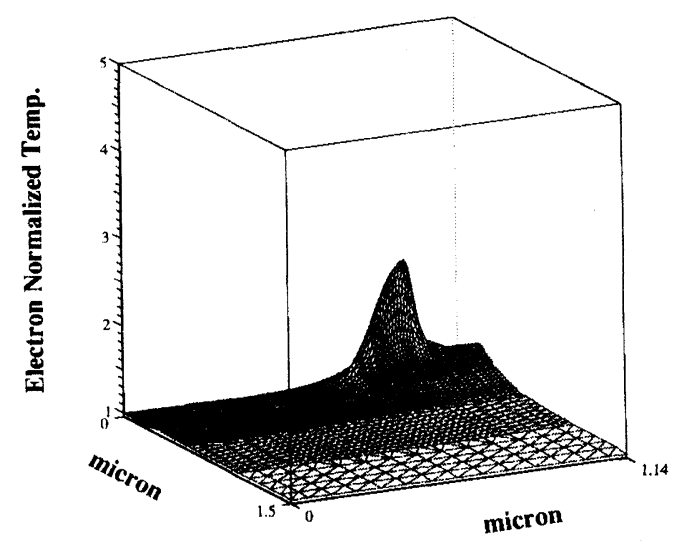

FIGURE 5 MOS device. Normalized temperature within the device at the beginning of the ramp

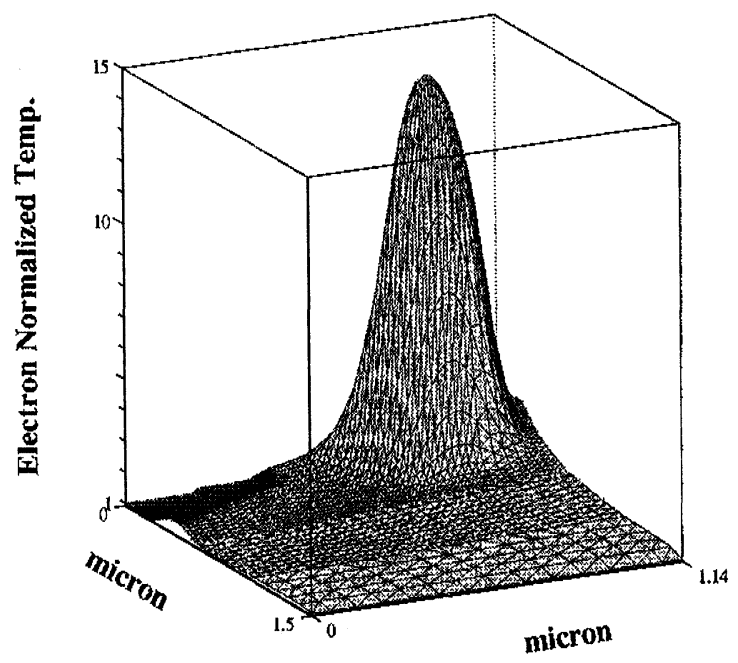

FIGURE 6 MOS device. Normalized temperature within the device at the end of the ramp 


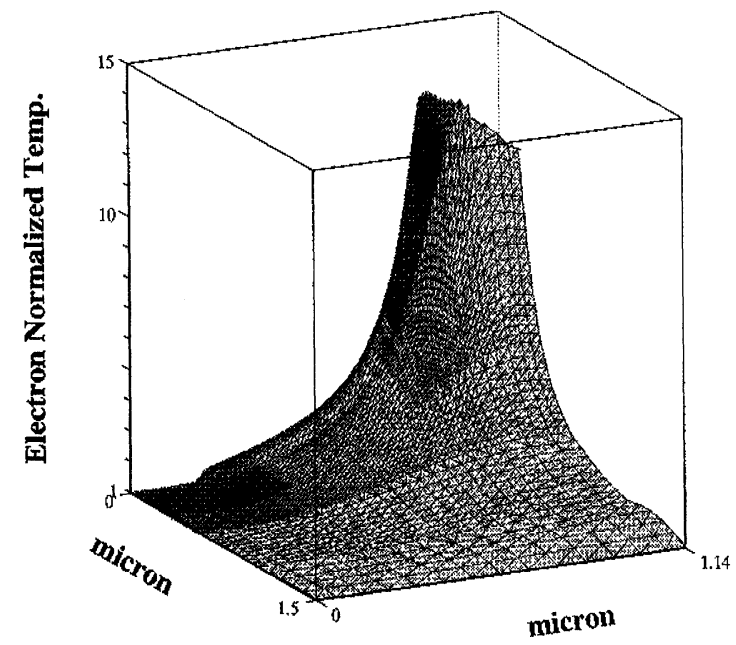

FIGURE 7 MOS device. Normalized temperature within the device at the end of the transient

malized temperature at the beginning of the ramp at $t=1 \mathrm{psec}$, i.e., when the ramp reaches $3 \mathrm{~V}$, and at the end of the transient. In the direction of the channel the results are qualitatively similar to those of the previous case: the temperature peak is initially localized in the channel region while, after the end the ramp, it starts spreading to eventually reach the steady-state profile of Fig. 7. In addition, thanks to the two-dimensional analysis, the spread of the energy from the surface to the bulk is also evident in the figure.

\section{References}

[1] A. Grubin et al., VLSI Microstructures Science, vol. 3.

[2] A. Grubin et al. VLSI Microstructures Science, vol. 10.

[3] A. Forghieri et al., IEEE T-CAD, vol. 7, no. 2, 1988, pp. 231242.

[4] A. Gnudi and F. Odeh, Proc. SISDEP, vol. 3, Bologna, 1988, pp. 387-390.

[5] G. Baccarani and M. Wordeman, Solid-State Electronics, vol. 28 , no. 4,1985 , pp. $407-416$.

\section{Biographies}

Luigi Colalongo was born in Bologna, Italy. $\mathrm{He}$ received the degree in Electrical Engineering from the University of Bologna in 1992 and, since then, he has been working at the Department of Electronics (DEIS) of the same University. He is engaged in an activity on the numerical simulation of semiconductor devices, with emphasis on amorphous and polycrys- talline devices. Actually he is working toward his $\mathrm{PhD}$ degree in Electrical Engineering at the University of Trento, Italy, in the field of chemical sensors.

Marina Valdinoci was born in Forli', Italy. She received the degree in Electrical Engineering from the University of Bologna in 1991. Since then, she has been working at the Department of Electronics (DEIS) of the University of Bologna, in the area of semiconductor devices modeling. Since 1993 she is working toward her $\mathrm{PhD}$ degree in Electrical Engineering. This activity is focused on the numerical simulation of amorphous and polycrystalline semiconductor devices.

Dr. Antonio Gnudi graduated in Electrical Engineering in 1983 at the University of Bologna, Italy, and received the Ph.D. degree in Electrical Engineering in 1989 at the same University. His dissertation involved numerical and physical aspects of semiconductor device modeling. From 1988 to 1990 he visited the IBM T.J. Watson Research Center, USA, where he was engaged in the modeling of submicrometer devices by means of higher-order moments of the Boltzmann Transport Equation, the so called "hydrodynamic" model. Since 1990 he is Teaching and Research Assistant at the Department of Electronics of the University of Bologna.

Massimo Rudan was born in Bologna, Italy, in 1949. He received a degree in Electrical Engineering in 1973 and a degree in Physics in 1976, both from the University of Bologna, Italy. He joined the Department of Electronics (DEIS) of the University of Bologna in 1975, where he investigated the physical properties of the MOS structures and the problems of analytical modelling of semiconductor devices. From 1978 he has been teaching an annual course of Quantum Electronics in the Faculty of Engineering of the same University. Since 1983 he has been working in a group involved in numerical analysis of semiconductor devices, acting as Task leader in a number of EEC-supported Projects in the area of CAD for VLSI. In 1986 he has been a visiting scientist, on a one-year assignment, at the IBM Thomas J. Watson Research Center at Yorktown Heights, NY. In 1990, he was appointed Full Professor of Microelectronics at the University of Bologna. 

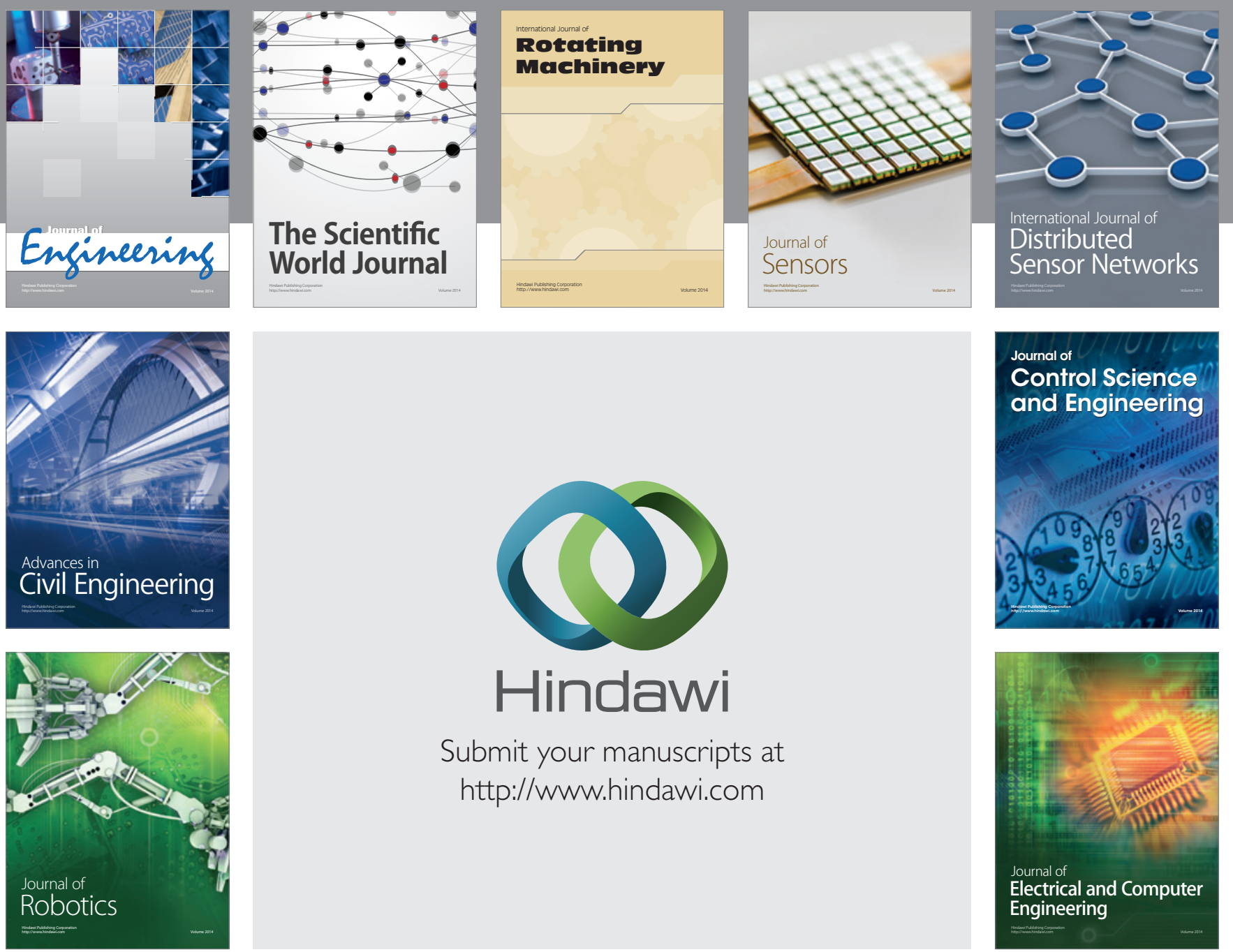

Submit your manuscripts at

http://www.hindawi.com
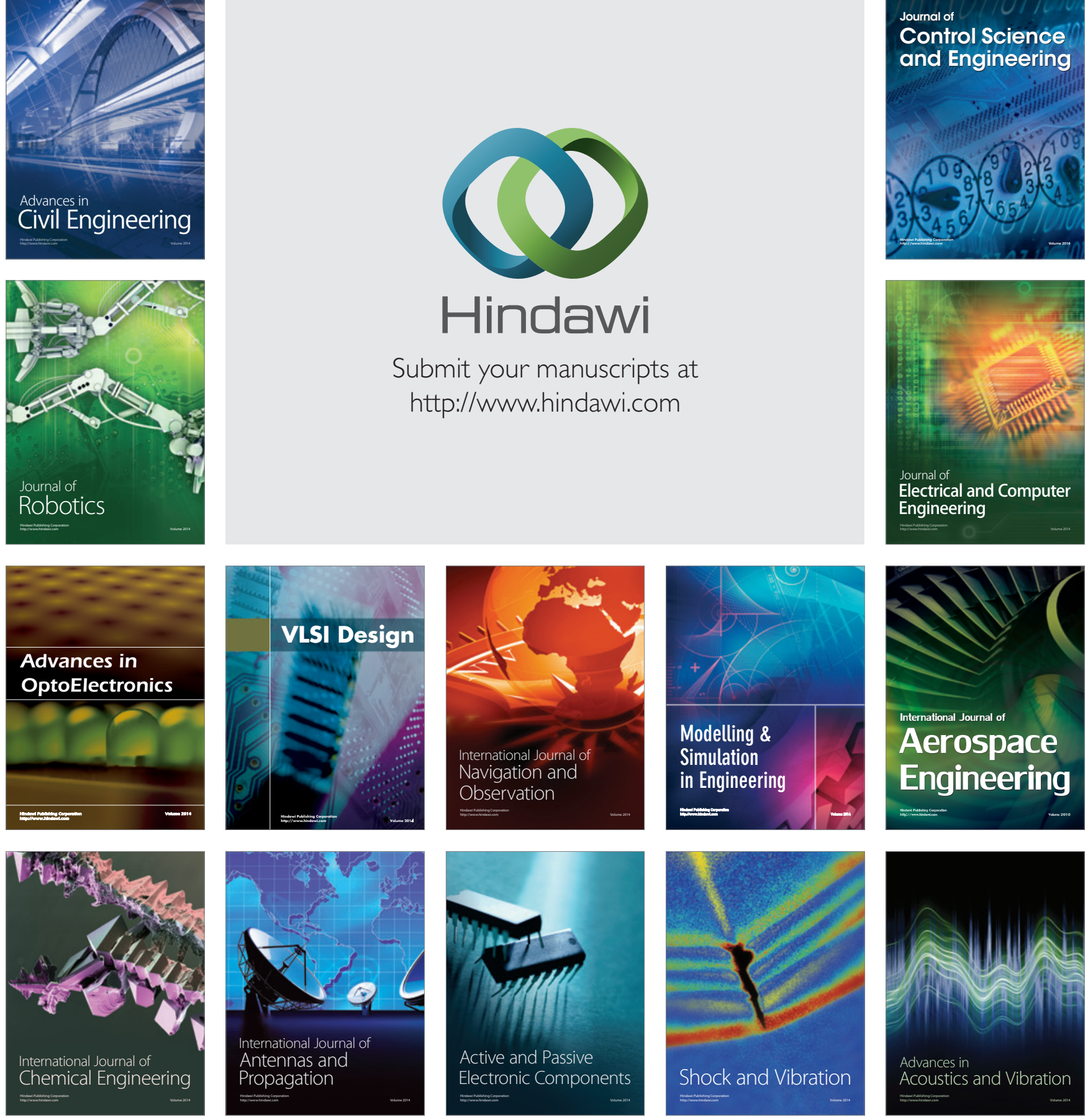